\title{
Defect Imaging and Structure Evolution in GST Films During In-situ Heating
}

\author{
Chanchal Ghosh ${ }^{1}$, Manish Singh ${ }^{1}$, John Watt ${ }^{2}$, Helena Silva ${ }^{1}$ and C Barry Carter ${ }^{3,4}$
}

${ }^{1}$ Department of Electrical \& Computer Engineering, University of Connecticut, Storrs, Connecticut, United States, ${ }^{2}$ Center for Integrated Nanotechnologies, Los Alamos, New Mexico, United States, ${ }^{3}$ Department of Chemical \& Biomolecular Engineering, University of Connecticut, Storrs, CT, United States, ${ }^{4}$ Center of Integrated Nanotechnologies, Sandia National Laboratories, Albuquerque, NM, USA

Phase-change materials that combine Ge, Sb and Te (known as GST materials) are one of the major contenders for future non-volatile data storage applications due to their difference in opto-electrical properties for the crystalline structure and the amorphous structure [1]. Phase-change electronic memory devices make use of the rapid and repeated switching between the phases through the application of electrical pulses. The difference in properties of these two phases are utilized to generate the " 0 " and " 1 " data series [2]. RESET and SET operations amorphize and crystallize a nanoscale volume of phase-change material that forms the active region of the device. During the RESET operation in the device application, an intense electrical pulse melts the crystalline phase which is then rapidly quenched giving an amorphous phase. In the SET operation, the amorphous phase is transformed into the crystalline phase with the application of a longer and less intense electrical pulse which provides the thermal energy to recrystallize the material.

During crystallization the amorphous $\mathrm{Ge}_{2} \mathrm{Sb}_{2} \mathrm{Te}_{5}$ phase initially nucleates into a metastable fcc structure at $\sim 150^{\circ}$ $\mathrm{C}$, which with a further increase in temperature, transforms into hcp phase at $\sim 250^{\circ} \mathrm{C}$ [3]. The switching nature in these materials greatly depends on the formation of intermediate phases and so a detailed understanding of the structure and chemistry of these phases will be helpful in assessing their operational behavior [4-5]. Previous reports also suggest that the pseudobinary compound $\mathrm{Ge}_{2} \mathrm{Sb}_{2} \mathrm{Te}_{5}$ can be considered as combinations of its binaries of GeTe and $\mathrm{Sb}_{2} \mathrm{Te}_{3}[6]$. In-situ heating inside the TEM equipped with a direct detection camera is considered most appropriate to study the phase transformations kinetics in this system [7-8].

In the present paper $\mathrm{Ge}_{2} \mathrm{Sb}_{2} \mathrm{Te}_{5}$ thin films of 10,20 and $30 \mathrm{~nm}$ were deposited on Protochips SiN substrates using magnetron sputtering and were imaged during in-situ heating using an Aduro 300 Protochips holder in an imagecorrected Titan environmental-TEM. To minimize the beam-induced damage and unintentional phase transformations, low-dose imaging was carried out using a direct-electron-detection Gatan K3-IS camera. To compare the intermediate phases of $\mathrm{Ge}_{2} \mathrm{Sb}_{2} \mathrm{Te}_{5}$ with its binaries, samples prepared from $99.99 \%$ purity GeTe and $\mathrm{Sb}_{2} \mathrm{Te}_{3}$ sputtering targets were also analyzed in the same TEM. The observed atomic-resolution phase-contrast images were analyzed with the aid of atomic structure modelling and multislice image simulation performed in CrystalMaker and Java based EMS (JEMS) software respectively [9].

In-situ heating of the $\mathrm{Ge}_{2} \mathrm{Sb}_{2} \mathrm{Te}_{5}$ thin film on the Protochips support shows the onset of the crystallization at a relatively lower temperature than observed with optical or electrical measurements. The nucleation of an intermediate structure at $\sim 100^{\circ} \mathrm{C}$ has been also observed during in-situ heating of the film. Figure 1(a) shows one such representative HRTEM micrograph of the phase. Interestingly the measured lattice spacing in this intermediate structure is $\sim 10 \AA$, which corresponds to a major phase in the binary $\mathrm{Sb}_{2} \mathrm{Te}_{3}$ alloy. Earlier studies showed chemical segregation of the $\mathrm{Ge}_{2} \mathrm{Sb}_{2} \mathrm{Te}_{5}$ films on Protochips into a $\mathrm{Ge}$ rich and Sb-Te rich phases during in-situ heating experiment and also the presence of oxygen along with $\mathrm{Ge}$ in the phase-separated domains. In order to analyze the structure and stoichiometry of the newly formed phases, the atomic-resolution HRTEM micrograph of $\mathrm{Sb}_{2} \mathrm{Te}_{3}$ binary along the [20] zone axis is shown in figure 1(b). A corresponding simulated thickness-defocus map of the $\mathrm{Sb}_{2} \mathrm{Te}_{3}$ binary in similar imaging conditions is shown in Figure 2. A detailed comparison of the intermediate structure in the heated $\mathrm{Ge}_{2} \mathrm{Sb}_{2} \mathrm{Te}_{5}$ films on Protochips and the $\mathrm{Sb}_{2} \mathrm{Te}_{3}$ phase confirms the similar nature of the two structures. The phase separation of in-situ heated $\mathrm{Ge}_{2} \mathrm{Sb}_{2} \mathrm{Te}_{5}$ films on Protochips into Ge and Sb-Te rich phases may be related to the reduction of the observed crystallization temperature.

This research is funded by NSF under award DMR-1710468. TEM investigation was carried out at CINT, an Office of Science User Facility operated for the U.S. DOE; Sandia National Labs is managed and operated by National Technology and Engineering Solutions of Sandia, LLC., a wholly owned subsidiary of Honeywell International, 
Inc., for the U.S. DOE's NNSA contract DE-NA-0003525. The views expressed here do not necessarily represent the views of the U.S. DOE or the U.S. Government. Los Alamos National Laboratory, an affirmative action equal opportunity employer, is managed by Triad National Security, LLC for the U.S. Department of Energy's NNSA, under contract 89233218CNA000001.

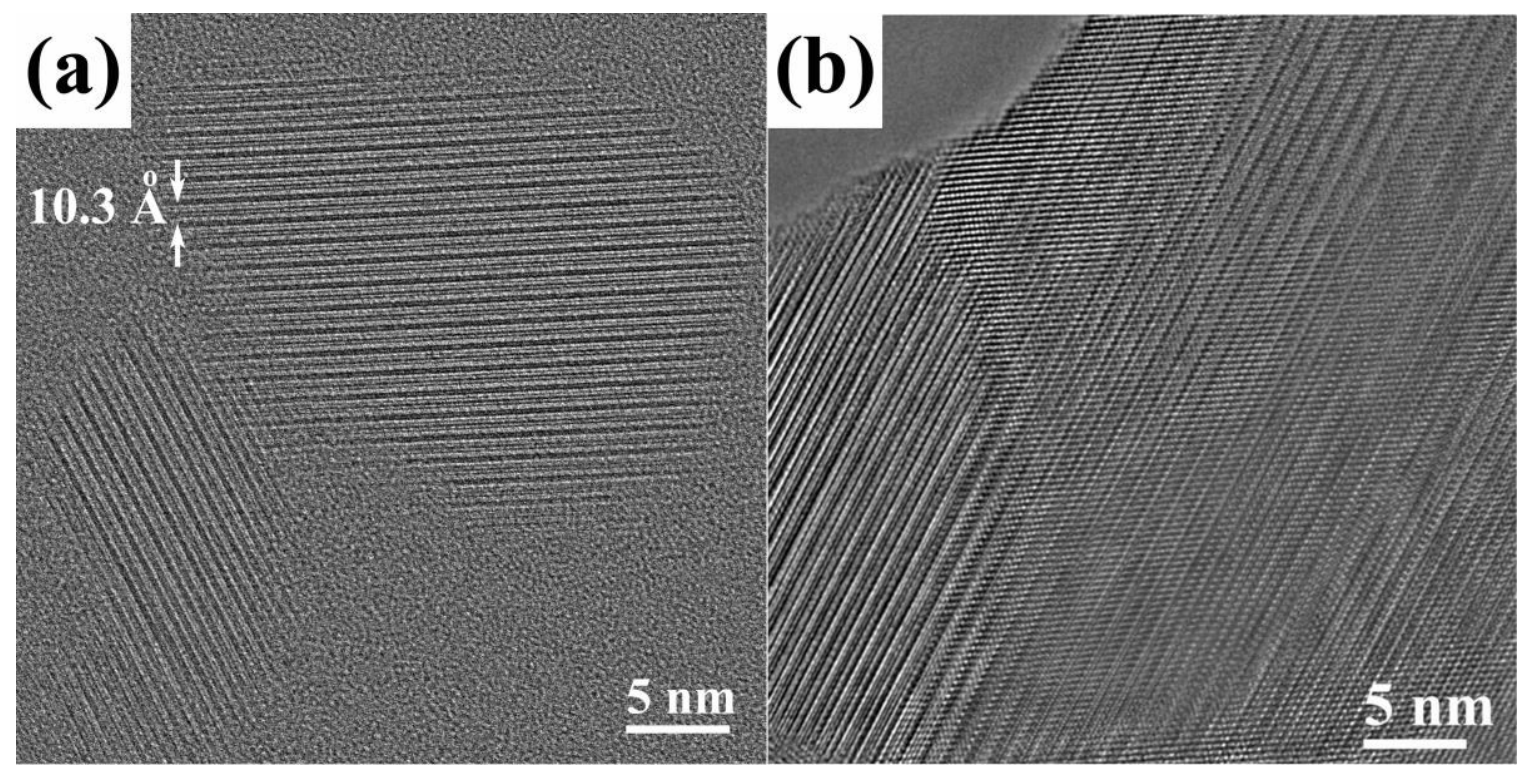

Figure 1. Atomic resolution phase contrast micrographs from (a) intermediate structure of Ge2Sb2Te5 thin films on Protochips during in-situ heating, and (b) Sb2Te3 crystallites along [20] zone axis.

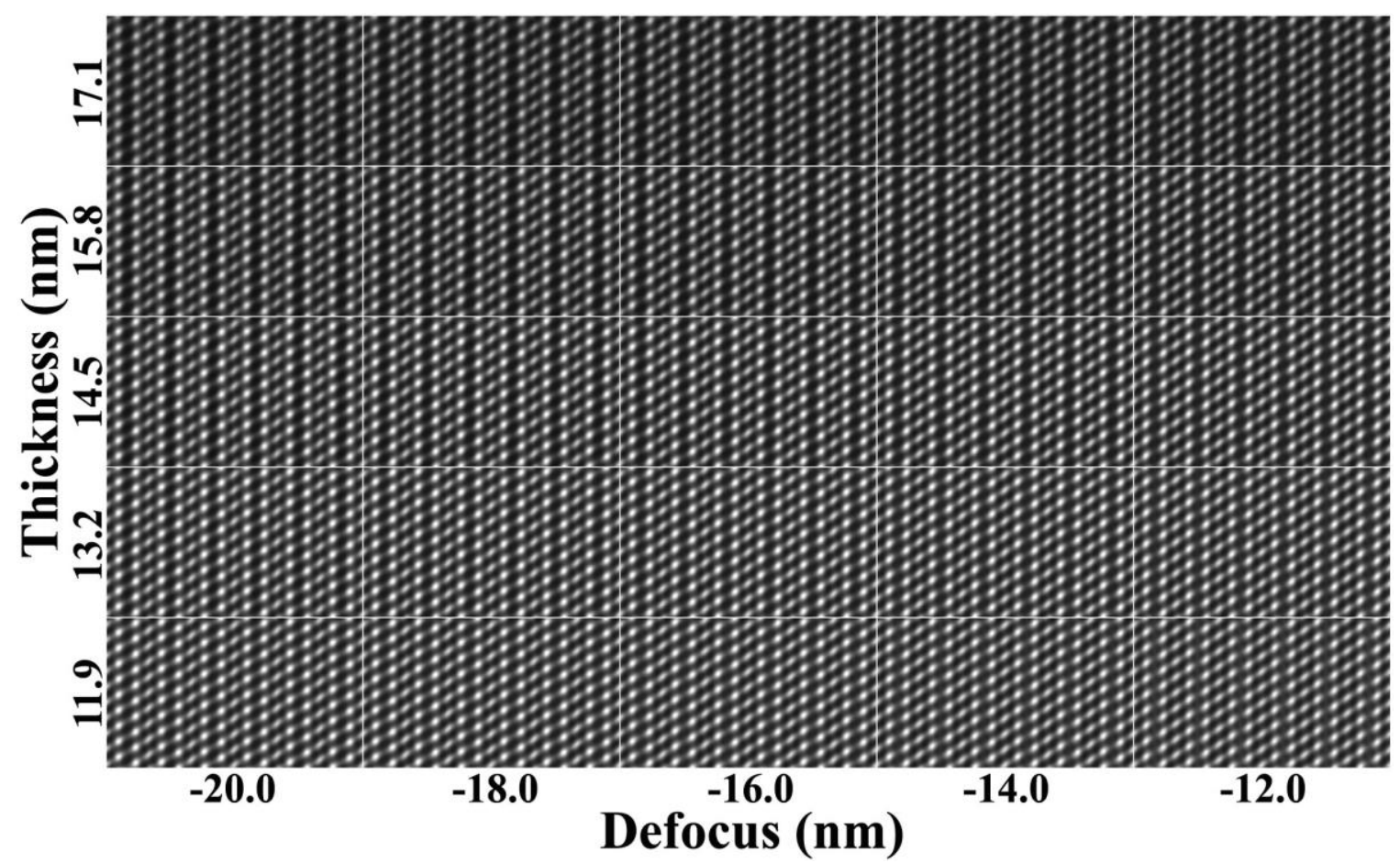

Figure 2. Thickness-defocus map of $\mathrm{Sb} 2 \mathrm{Te} 3$ phase along [20] zone axis using multislice image simulation algorithm in JEMS. 


\section{References}

[1] Hegedus J, Elliott SR (2008) Microscopic origin of the fast crystallization ability of Ge-Sb-Te phase-change memory materials. Nature Materials 7(5), 399-405

[2] Friedrich I, Weidenhof V, Njoroge W, Franz P, Wuttig M (2000) Structural transformations of Ge2Sb2Te5 films studied by electrical resistance measurements. Journal of Applied Physics 87, 4130-4134

[3] Tripathi S, Janish M, Dirisaglik F, Cywar A, Zhu Y, Jungjohann K, Silva H, Carter CB (2018) Phase-Change Materials; the Challenges for TEM. Microscopy and Microanalysis 24, S1, 1904-05.

[4] Lazzarini L, Rotunno E (2017) Crystal structure assessment of Ge-Sb-Te nanowires. Materials Science in Semiconductor Processing 65, 77-87

[5] Lotnyk A, Bernutz S, Sun X et al. (2016) Real-space imaging of atomic arrangement and vacancy layers ordering in laser crystallised $\mathrm{Ge}_{2} \mathrm{Sb}_{2} \mathrm{Te}_{5}$ phase change thin films. Acta Materialia 105, 1-8

[6] Zheng Y, Xia M, Cheng Y et al. (2016) Direct observation of metastable face centered cubic $\mathrm{Sb}_{2} \mathrm{Te}_{3}$ crystal. Nano Research 9(11), 3453-3462

[7] Williams DB, Carter CB (2009) Transmission electron microscopy- A textbook for materials science, $2^{\text {nd }}$ Ed. Springer, New York

[8] Carter CB, Williams DB (Eds.) (2016) Transmission electron microscopy: diffraction, imaging and spectrometry, Springer, Berlin Heidelberg, 17-80

[9] Stadelmann P (1987) EMS - a software package for electron diffraction analysis and HREM image simulation in materials science, Ultramicroscopy 21(2), 131-145 